\title{
Thermodynamic Effects of Replacements of Pro Residues in Helix Interiors of Maltose-Binding Protein
}

\author{
R.S. Prajapati, ${ }^{1}$ G.M. Lingaraju, ${ }^{1}$ Kiran Bacchawat, ${ }^{1}$ Avadhesha Surolia, ${ }^{1}$ and $\operatorname{Raghavan} \operatorname{Varadarajan}^{1,2 *}$ \\ ${ }^{1}$ Molecular Biophysics Unit, Indian Institute of Science, Bangalore, India \\ ${ }^{2}$ Chemical Biology Unit, Jawaharlal Nehru Center for Advanced Scientific Research, Bangalore, India
}

\begin{abstract}
Introduction of Pro residues into helix interiors results in protein destabilization. It is currently unclear if the converse substitution (i.e., replacement of Pro residues that naturally occur in helix interiors would be stabilizing). Maltose-binding protein is a large 370 -amino acid protein that contains 21 Pro residues. Of these, three nonconserved residues (P48, P133, and P159) occur at helix interiors. Each of the residues was replaced with Ala and Ser. Stabilities were characterized by differential scanning calorimetry (DSC) as a function of $\mathrm{pH}$ and by isothermal urea denaturation studies as a function of temperature. The P48S and P48A mutants were found to be marginally more stable than the wild-type protein. In the $\mathrm{pH}$ range of $5-9$, there is an average increase in $T_{m}$ values of $\mathrm{P48A}$ and $\mathrm{P} 48 \mathrm{~S}$ of $0.4^{\circ} \mathrm{C}$ and $0.2^{\circ} \mathrm{C}$, respectively, relative to the wild-type protein. The other mutants are less stable than the wild type. Analysis of the effects of such Pro substitutions in MBP and in three other proteins studied to date suggests that substitutions are more likely to be stabilizing if the carbonyl group i-3 or i-4 to the mutation site is not hydrogen bonded in the wild-type protein. Proteins 2003;53:863-871. ๑ 2003 Wiley-Liss, Inc.
\end{abstract}

Key words: mutant; stability; hydrogen bond

\section{INTRODUCTION}

Increasing protein stability through rationally designed mutations has been the goal of several protein engineering studies. Although there has been considerable progress in this area in recent years, it remains a challenging task. One approach is to identify potential destabilizing interactions in the wild-type protein and remove these by appropriate mutations. In an $\alpha$-helix, the amide proton of residue $\mathrm{i}$ is hydrogen bonded to the carbonyl oxygen of residue i-4. Pro residues lack an amide hydrogen and, hence, are typically not found in the hydrogen bonded, interior regions of $\alpha$-helices. There have been several studies that clearly show that introduction of a Pro residue into the interior of an $\alpha$-helix is destabilizing. ${ }^{1,2}$ However, there are comparatively fewer studies that have examined the thermodynamic effects of replacing potentially destabilizing Pro residues in interiors of naturally occurring $\alpha$-helices. This is in part because Pro residues are not commonly found at such locations. Although it is not common to find Pro in the interior of an $\alpha$-helix, this occurs with sufficient frequency $(14 \%)^{3}$ to make such replacements a potentially useful method of protein stabilization. Because Pro containing $\alpha$-helices can be identified through secondary structure prediction algorithms alone, Pro replacements could be a useful complement to more sophisticated structure-based methods of protein stabilization. Most protein stability studies have focused on small proteins because larger proteins do not show reversible unfolding. Small proteins typically have at most a single Pro in the interior of an $\alpha$-helix in the entire protein. This makes it difficult to examine multiple sites in the same protein.

In the present work we have characterized the effects of $\alpha$-helical Pro substitutions at three positions in maltosebinding protein (MBP). MBP is a 370-amino acid, twodomain protein found in the periplasm of Escherichia coli. It is involved in binding and transport of maltose. We have previously characterized the unfolding of the wild-type protein by chemical denaturation and differential scanning calorimetry (DSC). ${ }^{4,5}$ In both cases, unfolding is reversible, and the data fit well to a two-state model. High-resolution crystal structures of MBP in the maltose bound and free states are available. ${ }^{6,7}$ Thus, MBP is an ideal candidate for protein-folding studies. Unlike many smaller proteins, MBP contains 21 Pro residues distributed throughout the structure. It contains three Pro residues at positions 48,133 , and 159 , which are in the interior of $\alpha$-helices. These Pro residues are not conserved in MBP homologs. Pro was replaced by Ser and Ala at all three positions. The Ser substitution was chosen because Pro and Ser have similar hydrophobicities and volumes, and in a previous study, ${ }^{8}$ replacement of an $\alpha$-helical Pro by Ser resulted in protein stabilization. The hydroxyl group on the Ser chain is also capable of participating in additional hydrogen bonds with the backbone. Ala was chosen because it is a small, nonpolar residue with a high

Abbreviations: MBP, maltose-binding protein; EDTA, ethylenediaminetetraacetic acid; CGH10, $10 \mathrm{mM}$ each of citrate, glycine, and HEPES buffer; DSC, differential scanning calorimetry; GdnHCl, guanidine hydrochloride; WT, wild type.

\footnotetext{
Grant sponsors: DST and DBT (Government of India).

*Correspondence to: Raghavan Varadarajan, Molecular Biophysics Unit, Indian Institute of Science, Bangalore 560 012, India. E-mail: varadar@mbu.iisc.ernet.in
}

Received 3 January 2003; Accepted 16 April 2003 


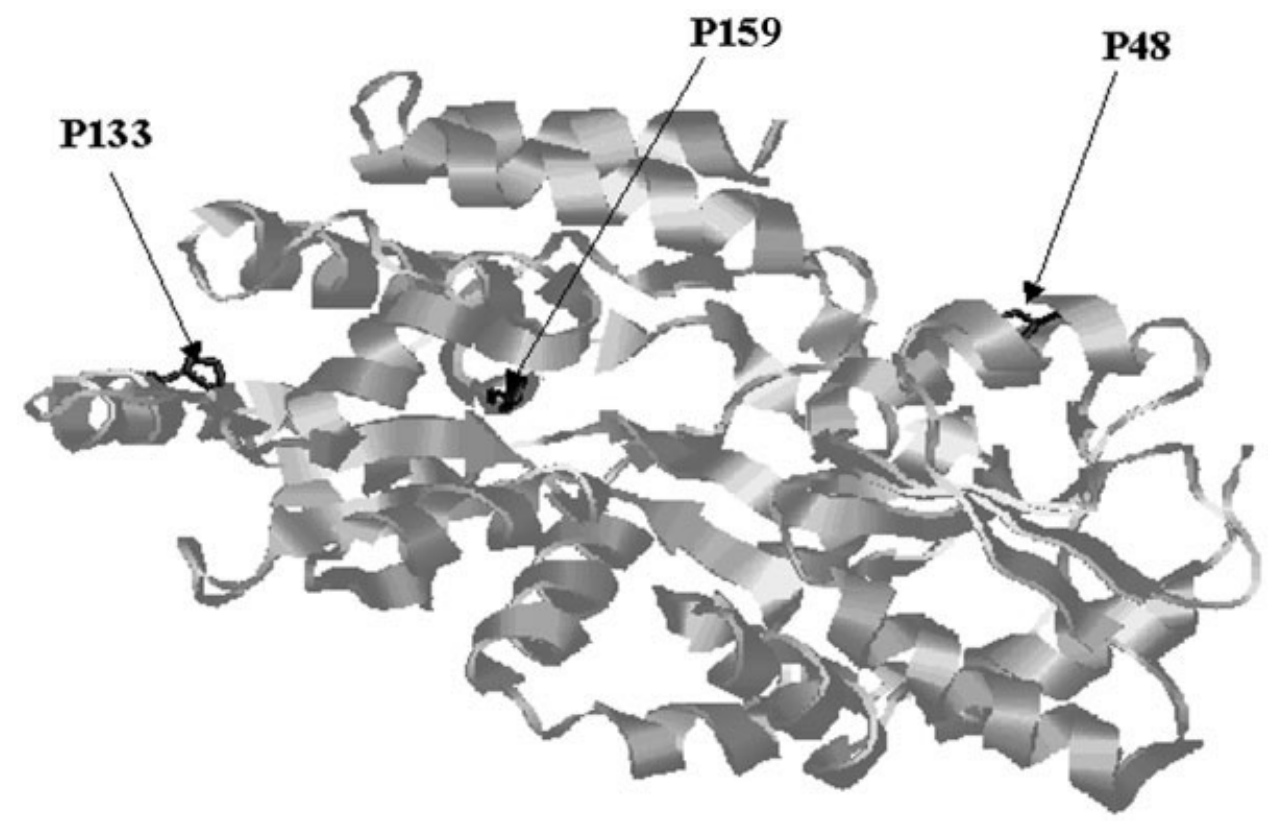

Fig. 1. Locations of the three Pro residues in the crystal structure of maltose-binding protein. The ribbon diagram was generated with the program RASTOP. ${ }^{25}$

helical propensity. Positions 48 and 133 are exposed, whereas position 159 is buried (Fig. 1). All six mutants were purified, and the unfolding thermodynamics were characterized.

\section{MATERIALS AND METHODS Mutagenesis, Expression, and Protein Purification}

The $E$. coli $K-12$ strain harboring the plasmid pPD1 was used as the source of MBP. The plasmid pPD1 containing the wild-type $E$. coli $M a l E$ gene and its $E$. coli host PD2 $8^{9,10}$ were provided by M. Hofnung. The gene was also cloned and expressed in the vector pMAL-p2 (New England Biolabs) plasmid for the overexpression of MBP using induction with $0.1 \mathrm{mM}$ 1PTG. pMALp2 is designed to create $\mathrm{C}$-terminal fusions between MBP and a cloned gene of interest. pBSKS ${ }^{+} \mathrm{MalE}^{11}$ and pMALp2 were both double digested with $B g l \mathrm{II}$ and HindIII. These enzymes cleave the $\mathrm{C}$-terminal region, which includes a stop codon for MalE. The resulting small fragment from $\mathrm{pBSKS}^{+} \mathrm{MalE}$ was ligated with the large fragment of pMALp2 to give pMALp2-MBP. This expresses full-length wild-type MBP under control of the tac promoter, in contrast to pPD1, which expresses the same protein using the malE promoter. The P133S and P159S mutants were constructed in pPD1, and the P48S and P159A mutants were constructed in pMALp2-MBP. The mutations were carried out by using a PCR-based site-directed mutagenesis strategy. The presence of each mutation was confirmed by sequencing the MalE gene. The mutant plasmid was transformed into the $E$. coli malE deletion strain pop-6590 for mutant protein expression. Mature MBP was isolated from $E$. coli cells with use of an osmotic shock procedure. ${ }^{4}$

\section{Isothermal, Equilibrium Unfolding of MBP as a Function of Temperature at Neutral pH}

Isothermal urea denaturation studies and data analysis were conducted in 0-7 M urea in CGH-10 buffer containing $150 \mathrm{mM} \mathrm{KCl}$ at $\mathrm{pH} 7.1$ as described previously. ${ }^{5}$ The protein concentration was $0.5 \mu \mathrm{M}$ for all denaturation studies. Protein concentrations were estimated as described. ${ }^{4}$ Denaturation experiments were conducted at various temperatures in the range of $10-45^{\circ} \mathrm{C}$ by incubating MBP in appropriate concentrations of urea until equilibria were established. Denaturant concentrations were calculated from measurements of the index of refraction. The values of $\Delta G^{\circ}$ and $m\left(m=\Delta G_{i}\right)$ at a given temperature were estimated according to the linear free energy model ${ }^{12,13}$ as described previously. ${ }^{5}$ All the data were analyzed by using Sigmaplot ${ }^{\mathrm{TM}}$ for Windows ${ }^{\mathrm{TM}}$ scientific graphing software.

\section{Differential Scanning Calorimetry}

DSC scans of the change in excess heat capacity of MBP as a function of temperature were measured by using the VP-DSC Microcalorimeter provided by Microcal ${ }^{\mathrm{TM}}$ Inc. DSC measurements were conducted by using a protein concentration of about $20 \mu \mathrm{M}$ as a function of $\mathrm{pH}$ in CGH-10 buffer using a scan rate of $90^{\circ} \mathrm{C} / \mathrm{h}$ as described previously. ${ }^{4}$ Scans were performed from 25 to $85^{\circ} \mathrm{C}$ for all the mutants. DSC data were fit to a two-state model in all cases.

\section{Structural Analysis}

The coordinates of thioredoxin (2trx), T4 lysozyme (3lzm, 1132) Fis (3fis, 1fip), and maltose-binding protein (1omp/ 11ls) were obtained from the protein data bank of the 
TABLE I. Unfolding Thermodynamic Parameters Obtained From Isothermal Denaturation Studies as a Function of Temperature ${ }^{\dagger}$

\begin{tabular}{|c|c|c|c|c|c|c|c|c|c|c|c|}
\hline \multirow[b]{2}{*}{$T$} & \multirow[b]{2}{*}{$-m$} & \multicolumn{2}{|c|}{ P133S } & \multicolumn{2}{|c|}{ P159A } & \multicolumn{2}{|c|}{ P159S } & \multicolumn{2}{|c|}{$\mathrm{P} 48 \mathrm{~S}$} & \multicolumn{2}{|l|}{ WT } \\
\hline & & $\Delta \Delta G^{\circ}$ & $\Delta C m$ & $\Delta \Delta G^{\circ}$ & $\Delta C m$ & $\Delta \Delta G^{\circ}$ & $\Delta C m$ & $\Delta \Delta G^{\circ}$ & $\Delta C m$ & $\Delta G^{\circ}$ & $\mathrm{Cm}$ \\
\hline 10 & $2.9 \pm 0.1$ & $-0.4 \pm 0.5$ & -0.1 & $-1.5 \pm 0.5$ & -0.5 & $-1.8 \pm 0.5$ & -0.6 & $-0.8 \pm 0.5$ & -0.2 & $9.9 \pm 0.4$ & 3.3 \\
\hline 14 & $3.9 \pm 0.1$ & $+1.5 \pm 0.7$ & +0.4 & $-0.7 \pm 0.7$ & -0.2 & $-1.9 \pm 0.7$ & -0.5 & $+0.5 \pm 0.7$ & +0.1 & $12.2 \pm 0.5$ & 3.1 \\
\hline 22 & $5.0 \pm 0.1$ & $0.0 \pm 0.7$ & 0.0 & $-1.0 \pm 0.7$ & -0.2 & $-1.7 \pm 0.6$ & -0.4 & $-0.3 \pm 0.7$ & -0.1 & $16.6 \pm 0.5$ & 3.3 \\
\hline 30 & $5.8 \pm 0.2$ & $-1.4 \pm 1.0$ & -0.2 & $-1.1 \pm 1.0$ & -0.2 & $-1.0 \pm 1.0$ & -0.2 & $-0.4 \pm 1.1$ & -0.1 & $18.9 \pm 0.8$ & 3.2 \\
\hline 37 & $5.6 \pm 0.2$ & $-0.3 \pm 0.7$ & -0.1 & $1.3 \pm 0.7$ & 0.2 & $-0.4 \pm 0.7$ & -0.1 & $0.4 \pm 0.7$ & +0.0 & $16.0 \pm 0.5$ & 2.8 \\
\hline
\end{tabular}

${ }^{\dagger}$ Change in parameters for mutants are indicated relative to values for WT. Units are as follows: $T\left({ }^{\circ} \mathrm{C}\right) ; \Delta \Delta G^{\circ}\left(\mathrm{kcal} \mathrm{mol}^{-1}\right) ; m\left(\mathrm{kcal} \mathrm{mol}{ }^{-1} \mathrm{M}^{-1}\right)$; $\Delta C m(\mathrm{M}), \Delta \Delta G^{\circ}=\Delta G^{\circ}$ (mutant) $-\Delta G^{\circ}(\mathrm{WT})$. Average values of $\Delta \Delta G^{\circ}$ are $-0.12,-0.60,-1.4$, and $-0.12 \mathrm{kcal} \mathrm{mol}^{-1}$ for P133S, P159A, P159S, and $\mathrm{P} 48 \mathrm{~S}$, respectively.

Research Collaboration for Structural Bioinformatics. ${ }^{14}$ Hydrogen bonds were analyzed with the program HBplus. $^{15}$

\section{RESULTS AND DISCUSSION Urea Denaturation Studies at Neutral pH}

A series of isothermal urea denaturation studies were conducted as a function of temperature for both the wild-type (WT) and four mutant proteins (Table I). Because DSC studies (see below) showed that the P133A and P48A mutants had similar stabilities to the corresponding P133S and P48S mutants, these were not characterized by urea unfolding. Urea was used instead of $\mathrm{GdnHCl}$ because transition with the latter denaturant are highly cooperative (characterized by very high $m$ values ${ }^{5}$ ). Therefore, it is technically difficult to obtain a large number of points in the transition region. Urea denaturation is also highly cooperative, ${ }^{5}$ but the $m$ value is somewhat lower (5 vs 12 kcal mol ${ }^{-1} \mathrm{M}^{-1}$ for $\mathrm{GdnHCl}$ ). Unfolding was monitored by measuring the fluorescence intensity at $337 \mathrm{~nm}$ as described previously. ${ }^{5}$

We previously showed that denaturation curves monitored by CD and fluorescence are superimposable for the wild-type protein and that denaturation data fit well to a two-state model. DSC studies (see below) were also consistent with two-state unfolding. In the present work, all urea denaturation data were fit to a two-state model as described previously. Because of the highly cooperative nature of the unfolding transition, $m$ values from individual melts are not reliable. Hence, a global fit was conducted by constraining the $m$ values of all mutants to be identical for each temperature at which isothermal denaturation studies were performed. The quality of the fits as well as the values of $\mathrm{Cm}$ obtained from these fits were virtually identical to those obtained without constraining $m$ values.

Table I and Figure 2 summarize the results of the isothermal denaturation studies. Values of $\Delta G^{\circ}$ and $\mathrm{Cm}$ for the wild-type protein determined in the present work are similar to those determined previously. ${ }^{5} \mathrm{WT}, \mathrm{P} 133 \mathrm{~S}$, and P48S are of comparable stability (average $\Delta \Delta G^{\circ}$ of $-0.12 \mathrm{kcal} / \mathrm{mol}$ ), whereas P159A and P159S are somewhat less stable than wild type with the average $\Delta \Delta G^{\circ}$ of -0.60 , $-1.4 \mathrm{kcal} / \mathrm{mol}$, respectively. These results are also in agreement with thermal stability measurements from DSC.

\section{Calorimetric Studies}

The thermal denaturation of the WT and all mutant proteins was characterized by DSC as previously described. ${ }^{4}$ The results of the DSC studies are summarized in Tables II and III, and a comparison of calorimetric scans for WT and the six mutants is shown in Figure 3. The enthalpies and $T_{m}$ values for the WT were similar to those described previously. ${ }^{4}$ The reversibility of thermal unfolding for the mutant proteins is similar to that reported previously for WT $\left(90 \%\right.$ at neutral $\left.\mathrm{pH}^{4}\right)$, and all scans fit well to a two-state model. The data show that P48S, P48A, and WT have very similar thermal stabilities over a wide $\mathrm{pH}$ range and are appreciably more stable than the other three mutant proteins. A paired $t$-test was used to show that the small increase in $T_{m}$ values at position 48 with respect to WT are statistically significant $(p=0.24$ and $p=$ 0.09 for $\mathrm{P} 48 \mathrm{~S}$ and $\mathrm{P} 48 \mathrm{~A}$, respectively). Values of $\Delta C_{p}$ were estimated from the temperature dependence of $\Delta H^{\circ}\left(T_{m}\right)$ in the $\mathrm{pH}$ range 6.5-10.5. Data at lower $\mathrm{pH}$ were not used because the reversibility of unfolding decreases drastically. $\Delta C_{p}$ values were estimated to be $3.9 \pm 0.6,7.0 \pm 2.9$, $5.9 \pm 0.3,4.1 \pm 0.5,5.5 \pm 0.5,5.4 \pm 0.5$, and $4.7 \pm 0.9$ $\mathrm{kcal}^{-1} \mathrm{~mol}^{-1} \mathrm{~K}^{-1}$ for the P133S, P133A, P159S, P159A, P48S, P48A, and WT, respectively (Fig. 4). Because the $T_{m}$ values of the mutant proteins are different, the measured values of $\Delta H^{\circ}\left(T_{m}\right)$ and $\Delta S\left(T_{m}\right)$ were extrapolated to a common reference temperature of $63^{\circ} \mathrm{C}$ (the $T_{m}$ of WT at $\mathrm{pH} 7.4$ ) by using the measured values of $\Delta C_{p}$. The changes in thermodynamic parameters in the mutants relative to those in WT at $63^{\circ} \mathrm{C}$ are summarized in Table III. The data show that with the exception of P48S and P48A, the other mutants are less stable than WT by about $2 \mathrm{kcal} / \mathrm{mol}$.

\section{Correlation of Observed Stabilities With Locations of Pro Residues in Structure}

The primary objective of this work was to determine if substitutions of Pro residues located in the interior of an $\alpha$-helix can lead to protein stabilization. The selection of such Pro residues is dependent on the criteria used to define the beginning and ends of the helix. Based on main-chain dihedral angle definitions, ${ }^{16}$ helices, which contain interior Pro residues, extend from 42-54, 128143, and 153-164 with interior Pro residues being located at positions 48, 133, and 159. If instead, hydrogen-bonding 


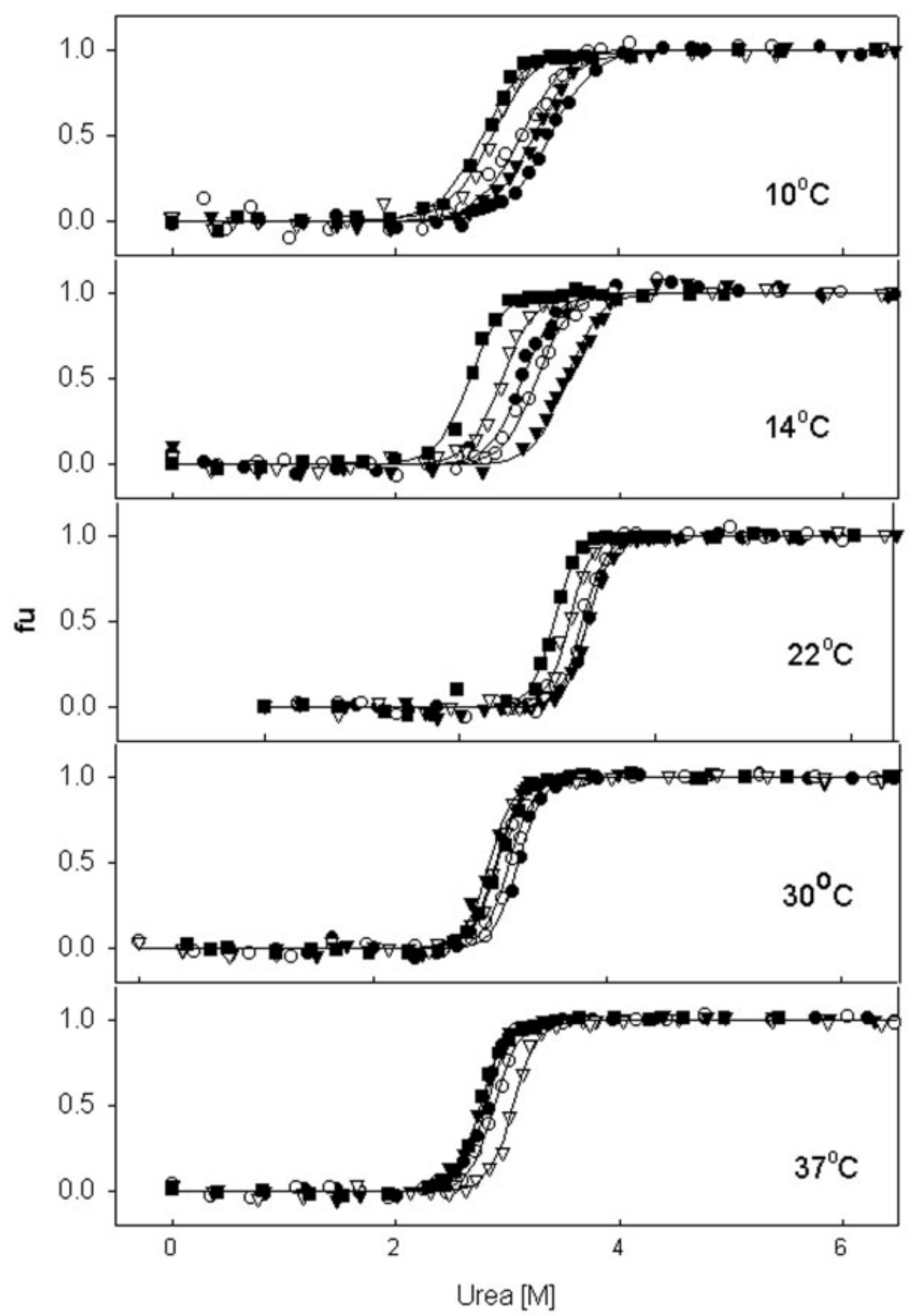

Fig. 2. Isothermal urea induced denaturation curves at $\mathrm{pH} 7.1$ : (A) $10^{\circ} \mathrm{C},(\mathrm{B}) 14^{\circ} \mathrm{C}$, (C) $22^{\circ} \mathrm{C}$, (D) $30^{\circ} \mathrm{C}$, and (E) $37^{\circ} \mathrm{C}$. The unfolding was monitored by monitoring change in fluorescence intensity at $342 \mathrm{~nm}$ with

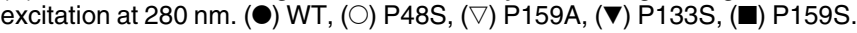

criteria are used to define the helical regions, ${ }^{17}$ the three above helices now extend from 43-52, 130-142, and 154163 , respectively. With these definitions, $\mathrm{P} 133$ is no longer in the interior but instead lies at the fourth position of the helix. The accessibilities ${ }^{18}$ of main-chain atoms are $10.6 \%, 11.3 \%$, and $0 \%$ for residues 48,133 , and 159 , and corresponding depths ${ }^{19}$ are $4.3,4.2$, and $8.6 \AA$, respectively. After Pro substitution, it is expected that a hydrogen bond will be formed between the new amide proton of the mutated residue (residue $i$ ) and the carbonyl oxygen of the residues at position i-4 and/or i-3. Therefore, it is of interest to examine the accessibility of the relevant carbonyl oxygens and whether they are hydrogen bonded in WT. This information is summarized in Table IV, which shows that the carbonyl oxygen of 
TABLE II. Thermodynamic Parameters for the Thermal Unfolding of WT and Mutant MBPs as a Function of pH Obtained from $\mathrm{DSC}^{\dagger}$

\begin{tabular}{|c|c|c|c|c|c|}
\hline Protein & $\mathrm{pH}$ & $T_{m}\left({ }^{\circ} \mathrm{C}\right)$ & $\Delta H^{\circ}(\mathrm{kcal} / \mathrm{mol})$ & $\Delta T_{m}\left({ }^{\circ} \mathrm{C}\right)$ & $\begin{array}{c}\text { Average } \\
\Delta \operatorname{Tm}\left({ }^{\circ} \mathrm{C}\right)\end{array}$ \\
\hline \multirow[t]{9}{*}{$\overline{\mathrm{WT}}$} & 6.5 & 65.3 & 238 & - & \\
\hline & 7.0 & 64.6 & 217 & & \\
\hline & 7.4 & 63.1 & 207 & - & \\
\hline & 8.0 & 61.3 & 185 & & \\
\hline & 8.5 & 60.5 & 178 & - & \\
\hline & 9.0 & 58.6 & 204 & & \\
\hline & 9.5 & 56.4 & 178 & - & \\
\hline & 9.9 & 52.4 & 165 & - & \\
\hline & 10.4 & 49.2 & 144 & - & \\
\hline \multirow[t]{9}{*}{ P48S } & 6.5 & 65.3 & 208 & 0.0 & 0.2 \\
\hline & 7.0 & 64.9 & 212 & 0.3 & \\
\hline & 7.4 & 63.7 & 211 & 0.6 & \\
\hline & 8.0 & 61.8 & 193 & 0.5 & \\
\hline & 8.5 & 60.4 & 180 & -0.1 & \\
\hline & 9.0 & 58.9 & 173 & 0.3 & \\
\hline & 9.5 & 56.0 & 162 & -0.4 & \\
\hline & 9.9 & 52.1 & 150 & -0.3 & \\
\hline & 10.4 & 49.7 & 116 & 0.5 & \\
\hline \multirow[t]{6}{*}{ P133S } & 6.5 & 62.0 & 212 & -3.3 & -4.7 \\
\hline & 7.4 & 59.7 & 205 & -3.4 & \\
\hline & 8.5 & 56.9 & 183 & -3.6 & \\
\hline & 9.5 & 51.3 & 152 & -5.1 & \\
\hline & 9.9 & 46.7 & 144 & -5.7 & \\
\hline & 10.4 & 42.1 & 139 & -7.1 & \\
\hline \multirow[t]{6}{*}{ P159S } & 6.5 & 61.2 & 199 & -4.1 & -4.4 \\
\hline & 7.4 & 59.5 & 184 & -3.6 & \\
\hline & 8.5 & 57.6 & 179 & -2.9 & \\
\hline & 9.5 & 50.3 & 129 & -6.1 & \\
\hline & 9.9 & 48.0 & 114 & -4.4 & \\
\hline & 10.4 & 44.0 & 100 & -5.2 & \\
\hline \multirow[t]{8}{*}{ P48A } & 6.5 & 65.5 & 219 & 0.2 & 0.4 \\
\hline & 7.0 & 64.1 & 208 & -0.5 & \\
\hline & 7.4 & 63.9 & 206 & 0.8 & \\
\hline & 8.0 & 62.6 & 195 & 1.3 & \\
\hline & 8.5 & 60.4 & 179 & -0.1 & \\
\hline & 9.0 & 59.2 & 162 & 0.6 & \\
\hline & 9.5 & 56.6 & 159 & 0.2 & \\
\hline & 9.9 & 53.0 & 137 & 0.6 & \\
\hline \multirow[t]{5}{*}{ P133A } & 6.5 & 63.0 & 235 & -2.3 & -0.5 \\
\hline & 7.4 & 62.5 & 182 & -0.6 & \\
\hline & 8.5 & 60.6 & 166 & 0.1 & \\
\hline & 9.5 & 56.4 & 148 & 0.1 & \\
\hline & 9.9 & 51.9 & 142 & 0.5 & \\
\hline \multirow[t]{6}{*}{ P159A } & 6.5 & 62.1 & 205 & -3.2 & -4.1 \\
\hline & 7.4 & 60.1 & 195 & -3.0 & \\
\hline & 8.5 & 56.9 & 187 & -3.6 & \\
\hline & 9.5 & 52.5 & 168 & -3.9 & \\
\hline & 9.9 & 49.0 & 135 & -3.4 & \\
\hline & 10.4 & 41.7 & 127 & -7.5 & \\
\hline
\end{tabular}

All data were fit to a two-state model.

${ }^{\dagger}$ Approximate errors are $\pm 0.1^{\circ} \mathrm{C}$ for $T_{m}$ and $5 \%$ for $\Delta H^{\circ}$.

residue 130 is highly exposed, whereas the carbonyl's of the other relevant residues are all buried.

All relevant carbonyl oxygens are hydrogen bonded in WT MBP, and this may be the primary reason for the lack of stabilization seen on mutation of the various Pro residues. In $\mathrm{P} 48$ mutants, carbonyls at both i-3 and i- 4 are
TABLE III. Changes in Thermodynamic Unfolding Parameters at $63^{\circ} \mathrm{C}$, pH 7.4, for MBP Mutants Relative to WT

\begin{tabular}{lccc}
\hline Protein & $\begin{array}{c}\Delta \Delta H^{\circ}\left(63^{\circ} \mathrm{C}\right) \\
(\mathrm{kcal} / \mathrm{mol})\end{array}$ & $\begin{array}{c}T \Delta \Delta S\left(63^{\circ} \mathrm{C}\right) \\
(\mathrm{kcal} / \mathrm{mol})\end{array}$ & $\begin{array}{c}\Delta \Delta G\left(63^{\circ} \mathrm{C}\right) \\
(\mathrm{kcal} / \mathrm{mol})\end{array}$ \\
\hline WT & - & - & - \\
P48S & 1.2 & 0.9 & 0.3 \\
P133S & 11.3 & 13.4 & -2.1 \\
P159S & -1.7 & 0.4 & -2.1 \\
P48A & -5.3 & -5.8 & 0.5 \\
P133A & -21.8 & -21.5 & -0.3 \\
P159A & 0.3 & 2.1 & -1.8 \\
\hline
\end{tabular}

hydrogen bonded in the wild-type structure [to side-chains of Gln 49 (i-3) and Tyr 70 and Arg 66 (i-4)]. Hence, although the residue at $\mathrm{i}-3$ is well positioned (Table IV) for forming an additional hydrogen bond with the amide hydrogen at position 48, the resulting hydrogen bond(s) are likely to be only marginally stabilizing. Analysis of the wild-type structure suggests several possible causes for the lack of stabilization seen in the P133 mutants. First, residues 129-141 all have main-chain dihedral angles in the right-handed helical region, with the exception of residue 131, which has phi and psi angles of -96 and 2 , respectively. This introduces a distortion into the $\mathrm{N}$ terminus of the helix. Consequently, as described above, residue 133 is not an interior residue if helices are defined according to hydrogen-bonding criteria. The carbonyl oxygens of residues 129 (i-4) and 130 (i-3) are located at distances of 4.7 and $4.5 \AA$, respectively, from the nitrogen atom of 133. The carbonyl oxygen at residue 129 is hydrogen bonded to nitrogen atom 132. The carbonyl at residue 130 is hydrogen bonded to a water molecule. Because this carbonyl is highly exposed, it probably forms additional hydrogen bonds with other disordered water molecules. Consequently, the amide hydrogen that would result from the P133 mutations would not have suitably positioned acceptors available. Although it is possible that conformational rearrangement of the acceptor carbonyl might result in formation of a hydrogen bond in the mutant protein, the stabilization relative to the wild type would still be small relative to the situation where the acceptor carbonyl was not hydrogen bonded in the wild type protein.

Because position 159 is fully buried, introduction of polar Ser side-chain is expectedly more destabilizing than the corresponding Ala substitution. In the mutants at position 159, the carbonyl residues at both 155 and 156 are fully buried. Although the carbonyl at position 155 is hydrogen bonded to an internal water molecule, no hydrogen bond appeared to be present in the vicinity of $\mathrm{O} 156$ in the structure of unliganded MBP (PDB code 1omp). Hence, the lack of stability in the corresponding Pro mutants at position 159 was initially puzzling. However, very recently $^{20}$ another structure of unliganded MBP (PDB code 1lls) was reported, and in this structure an additional water molecule with a low B-factor of $19 \AA^{2}$ was found at a distance of $2.9 \AA$ from O156. A similar water molecule is also found in the structure of maltose-bound MBP (PDB 


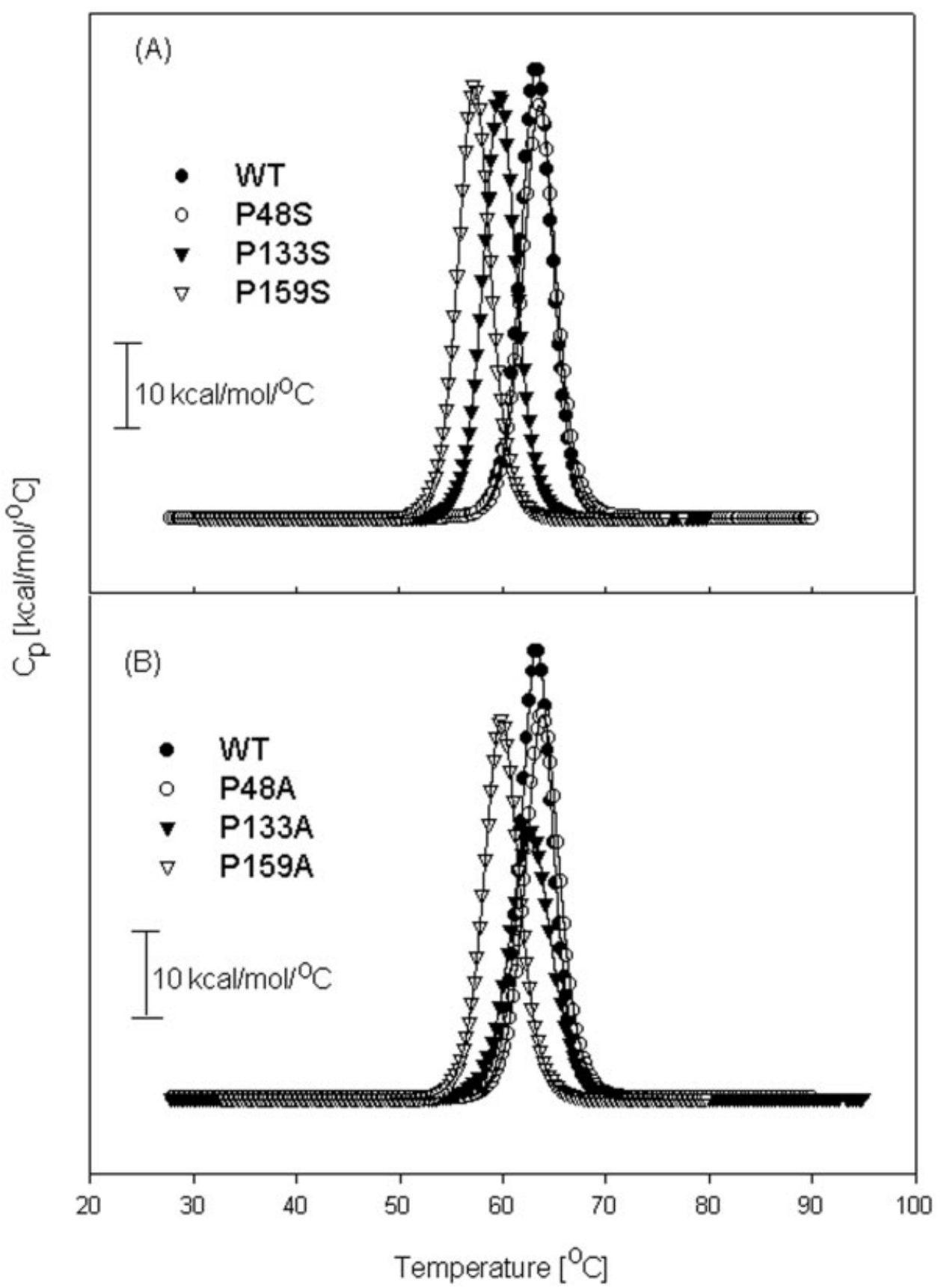

Fig. 3. Baseline subtracted DSC scans of MBP mutants at a concentration of $30 \mu \mathrm{M}, \mathrm{pH} 7.4$. The scan rate was $90^{\circ} \mathrm{C} / \mathrm{h}$. Data points are shown as symbols, and two- state fits to the data are shown as thin solid lines.

code lanf), suggesting that it is not an artefact of refinement.

To examine other factors that might contribute to lack of stabilization in the P159A and P159S mutations, an analysis was conducted of structural changes that occur in previously studied replacements of Pro in helix interiors. Crystal structures are available for substitutions of P61, $\mathrm{P} 86$, and $\mathrm{P} 40$ in Fis protein, T4 lysozyme, and $E$. coli thioredoxin, respectively. An analysis of dihedral angle changes in the P61A (1FIP) and P86S (1L32) mutants of the above two proteins revealed that the largest changes in phi and psi occurred from position $\mathrm{i}+1$ to $\mathrm{i}-3$ in each case. In MBP, residue 159 and its surrounding residues are deeply buried (Table IV), unlike the situation for residues 48 and 133 . The accessibilities of residues $156-160$ are 9 , $1,0,1$, and $1 \%$, respectively. Therefore, it is possible that the high degree of burial of residue 159 and its surroundings make it difficult for rearrangements that would facilitate hydrogen bond formation to occur on mutation. In contrast, at position 48 , because both $\mathrm{P} 48$ and its surrounding residues have high accessibility, dihedral angle adjustments to accommodate the mutation and to 


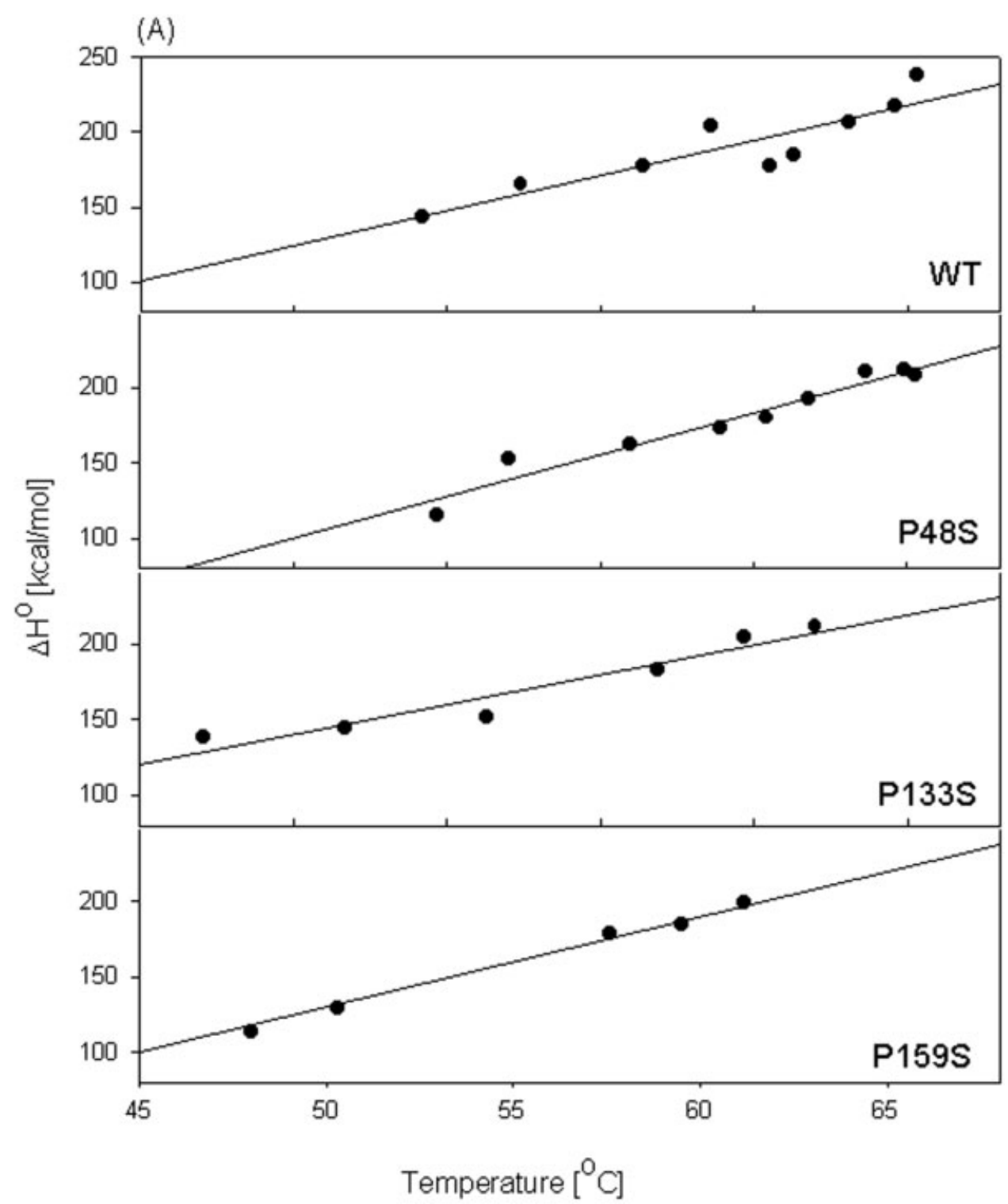

Fig. 4. $\Delta C_{p}$ estimation from the linear dependence of $\Delta H_{m}$ on $T_{m}$. The values of $\Delta C_{p}$ obtained from the slopes of these plots are $4.7 \pm 0.9,5.5 \pm 0.5,5.4 \pm 0.5,3.9 \pm 0.6,7.0 \pm 2.9,5.9 \pm 0.3$, and $4.1 \pm 0.5$ $\mathrm{kcal}^{-1} \mathrm{~mol}^{-1} \mathrm{~K}^{-1}$ for WT, P48S, P48A P133S, P133A, P159S, and P159A, respectively.

TABLE IV. Structural Parameters for Pro Residues Situated in the Helix Interior of Four Proteins

\begin{tabular}{|c|c|c|c|c|c|c|c|}
\hline$\underline{\text { Protein }}^{\mathrm{a}}$ & Residue (i) & Accessibility (\%) & Depth & $\begin{array}{c}\mathrm{ASA}\left(\AA^{2}\right) \text { of Os at } \\
\mathrm{i}-4 / \mathrm{i}-3\end{array}$ & $\begin{array}{c}\text { Distance }(\AA) \\
\mathrm{N}_{\mathrm{i}}-\mathrm{O}_{\mathrm{i}-4}\end{array}$ & $\begin{array}{c}\text { Distance }(\AA) \\
\mathrm{N}_{\mathrm{i}}-\mathrm{O}_{\mathrm{i}-3}\end{array}$ & $\begin{array}{c}\text { No. of } \\
\mathrm{H} \text { bonds for Os } \\
\text { at i-4/i-3 }\end{array}$ \\
\hline MBP & $\mathrm{P} 48 \mathrm{~S}$ & 25 & 4.1 & $0 / 0.7$ & 4.6 & 3.9 & $2 / 1$ \\
\hline MBP & P133 & 26 & 4.0 & $0 / 18$ & 4.7 & 4.5 & $1 / 1$ \\
\hline MBP & P159 & 0.8 & 9.0 & $0.2 / 1.2$ & 4.8 & 4.3 & $1 / 1^{\mathrm{c}}$ \\
\hline T4 Lysozyme & P86 & 41 & 3.8 & $11 / 5$ & 4.7 & 4.6 & $0 / 1$ \\
\hline Fis & P61 & 1.3 & 3.6 & $0 / 0$ & 4.5 & 3.9 & $0 / 1$ \\
\hline Thioredoxin & $\mathrm{P} 40$ & 56 & 3.7 & $0 / 0.4$ & 4.6 & 4.5 & $1 / 0$ \\
\hline
\end{tabular}

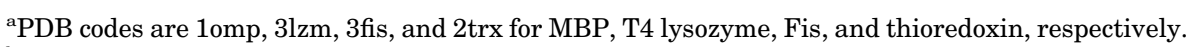

${ }^{\mathrm{b}}$ carbonyl oxygen atom.

${ }^{\mathrm{c}}$ Hydrogen bond to a water molecule detected in PDB codes IIIs (unliganded MBP) and lanf (maltose bound MBP).

form a main-chain hydrogen bond involving the amide of residue 48 are possible. The stability effects appear to be 


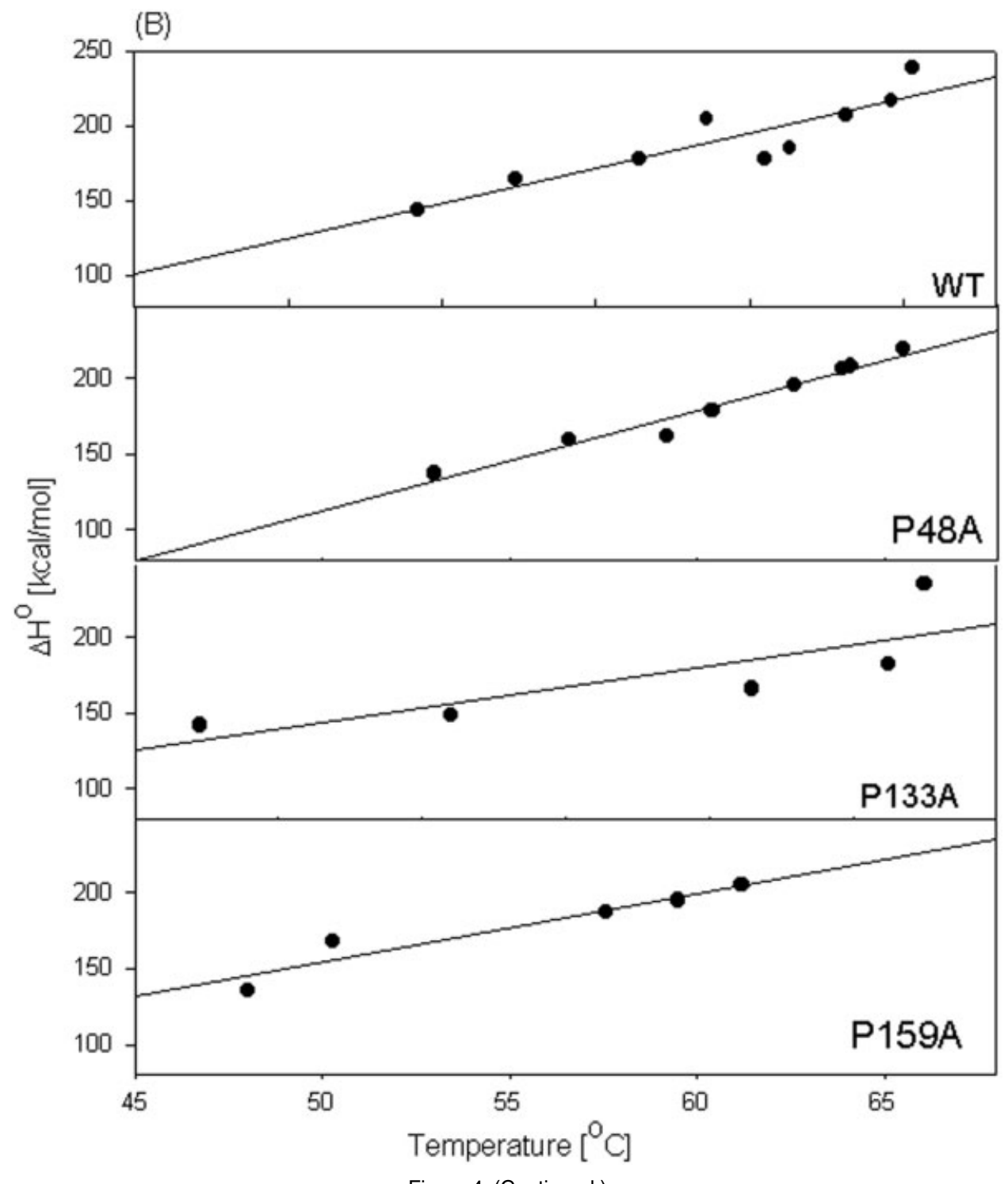

Figure 4. (Continued.)

dominated by hydrogen-bonding effects. The intrinsic secondary structural preferences of the substituted residue do play a minor role as can be seen from the small increase in $T_{m}$ values of the Ala substitutions at positions 48 and 133 .

\section{Comparisons With Previous Mutational Studies}

Before the present work, there were three mutational studies of Pro residue replacements at helix interiors. These involved P86S in T4 lysozyme ${ }^{21} \mathrm{P} 61 \mathrm{~A}$ in $E$. coli fis protein $^{22}$ and $\mathrm{P} 40 \mathrm{~S}$ in $E$. coli thioredoxin. ${ }^{8,23}$ In $\mathrm{T} 4$ lyzozyme, P86 occurs in a short surface helix that extends from residues 82-90. P86 was substituted by a number of other residues. However, no increase in $T_{m}$ of any of the mutants was observed. ${ }^{21} \mathrm{P} 61$ occurs in the interior of a long helix that extends from residues $50-70$ of Fis protein. The P61A mutant shows an increase in $T_{m}$ of about $15^{\circ} \mathrm{C}$ relative to the wild-type protein. $\mathrm{P} 40$ occurs in the center of a long helix that extends from residues $32-48$ in $E$. coli thioredoxin. The P40S mutant shows an increase in stability of $1-1.5 \mathrm{kcal} / \mathrm{mol}$ in the temperature range of $280-330$ $\mathrm{K}$. The only unsatisfied main-chain hydrogen bonds in the relevant helices of the wild-type proteins occur at the carbonyls of either position i-4 (in T4 lysozyme and Fis) or i-3 (in Trx). These carbonyl groups are buried in the case of Trx and Fis but are exposed in the case of T4 lysozyme (Table IV). The crystal structures of all three mutant proteins show that the amide of residue $i$ (which was originally Proline) forms the expected hydrogen bond with the carbonyl of residue i-4 (in T4 lysozyme) or both i-3 and i-4 (in Fis and Trx). The lack of stabilization seen in the P86S mutant of T4 lysozyme is probably because the exposed carbonyl group in the wild-type protein is hydrogen bonded to water molecules. Although no water molecules are seen within hydrogen-bonding distance of $\mathrm{O} 82$ in the crystal structure of wild-type T4 lysozyme, it is likely that these are present but disordered. In support of 
this, in the P86S mutant of T4 lysozyme, a water molecule is found at a distance of $3 \AA$ from O82. Additional hydrogen bonds due to solvent rearrangements around the site of mutation were also observed in the crystal structure of the P40S mutant of $E$. coli thioredoxin. ${ }^{23}$

Replacement of a helical Pro residue can have a stabilizing effect due to formation of an additional intrahelical hydrogen bond. Because Pro is a rigid residue, its substitution may also destabilize the protein by increasing the conformational entropy of the unfolded state. A recent analysis ${ }^{24}$ also showed that the $\mathrm{CD}$ atom of Pro could form a $\mathrm{C}-\mathrm{H}-\mathrm{O}$ hydrogen bond with residues at positions i-3 or i- 4 . The data in Table IV suggest that the following criteria (in decreasing order of importance) may be taken into account when choosing sites for Pro substitutions at interior helical positions. 1) The carbonyl group located at position i-3 or i-4 to the mutation should be relatively buried and not be hydrogen bonded in the wild-type structure (this means conventional hydrogen bonds, not the weaker $\mathrm{C}-\mathrm{H}-\mathrm{O}$ hydrogen bonds). 2) The Pro that is to be mutated as well as its surrounding residues should not be deeply buried in the structure. In addition, it is desirable that the mutations should not be made at highly conserved positions because conserved Pro residues are likely to be structurally and functionally important. For partially exposed Pro residues, rearrangements to accommodate the mutation and to form a new main-chain hydrogen bond are feasible. The mutational data also suggest that Ala may be a slightly better choice than Ser for substitution of Pro in helix interiors. Further mutational studies in other protein systems are required to confirm the validity of these suggestions. Unfortunately, it is not common to find interior helical Pro residues that satisfy both the above criteria. Even in MBP, a large protein with as many as 21 Pro residues, there were only three Pro residues that occurred at a helix interior, and in no case was it possible to achieve appreciable stabilization by mutation of these residues. This is likely to be due to the fact that in all three cases, carbonyl groups i-3 and i- 4 to the mutation were already hydrogen bonded either to other amino residues in the structure or to internal water molecules.

\section{ACKNOWLEDGMENTS}

This work was supported by grants from the Department of Science and Technology and the Department of Biotechnology (Government of India) to R.V. R.V. is a recipient of a Swarnajayanthi Fellowship (Government of India) and is a Senior Research Fellow of the Wellcome Trust. All DNA sequencing was conducted at the DNA Sequencing Facility, IISc. The VP-DSC is funded by DST to AS under the IRPHA program.

\section{REFERENCES}

1. Sauer UH, San DP, Matthews BW. Tolerance of T4 lysozyme to proline substitutions within the long interdomain alpha-helix illustrates the adaptability of proteins to potentially destabilizing lesions. J Biol Chem 1992;267:2393-2399.

2. Gray TM, Arnoys EJ, Blankespoor S, Born T, Jagar R, Everman R, Plowman D, Stair A, Zhang D. Destabilizing effect of proline substitutions in two helical regions of T4 lysozyme: leucine 66 to proline and leucine 91 to proline. Protein Sci 1996;5:742-751.
3. Gunasekaran K, Nagarajaram HA, Ramakrishnan C, Balaram P Stereochemical punctuation marks in protein structures: glycine and proline containing helix stop signals. J Mol Biol 1998;275:917-932.

4. Ganesh C, Shah AN, Swaminathan CP, Surolia A, Varadarajan R. Thermodynamic characterization of the reversible, two-state unfolding of maltose binding protein, a large two-domain protein. Biochemistry 1997;36:5020-5028.

5. Sheshadri S, Lingaraju GM, Varadarajan R. Denaturant mediated unfolding of both native and molten globule states of maltose binding protein are accompanied by large deltaCp's. Protein Sci 1999;8:1689-1695.

6. Spurlino JC, Lu GY, Quiocho FA. The 2.3-A resolution structure of the maltose-or maltodextrin-binding protein, a primary receptor of bacterial active transport and chemotaxis. J Biol Chem 1991;266: 5202-5219.

7. Sharff AJ, Rodseth LE, Spurlino JC, Quiocho FA. Crystallographic evidence of a large ligand-induced hinge-twist motion between the two domains of the maltodextrin binding protein involved in active transport and chemotaxis. Biochemistry 1992;31: 10657-10663.

8. Chakrabarti A, Srivastava S, Swaminathan CP, Surolia A, Varadarajan R. Thermodynamics of replacing an alpha-helical Pro residue in the P40S mutant of Escherichia coli thioredoxin. Protein Sci 1999;8:2455-2459.

9. Duplay P, Bedouelle H, Fowler A, Zabin I, Saurin W, Hofnung M. Sequences of the malE gene and of its product, the maltosebinding protein of Escherichia coli K12. J Biol Chem 1984;259: 10606-10613.

10. Martineau P, Szmelcman S, Spurlino JC, Quiocho FA, Hofnung M. Genetic approach to the role of tryptophan residues in the activities and fluorescence of a bacterial periplasmic maltosebinding protein. J Mol Biol 1990;214:337-352.

11. Ganesh C, Banerjee A, Shah A, Varadarajan R. Disordered $\mathrm{N}$-terminal residues affect the folding thermodynamics and kinetics of maltose binding protein. FEBS Lett 1999;454:307-311.

12. Schellman JA. The thermodynamic stability of proteins. Annu Rev Biophys Biophys Chem 1987;16:115-137.

13. Chen BL, Schellman JA. Low-temperature unfolding of a mutant of phage T4 lysozyme. I. Equilibrium studies. Biochemistry 1989; 28:685-691.

14. Berman HM, Westbrook J, Feng Z, Gilliland G, Bhat TN, Weissig $\mathrm{H}$, Shindyalov IN, Bourne PE. The Protein Data Bank. Nucleic Acids Res 2000;28:235-242.

15. McDonald IK, Thornton JM. Satisfying hydrogen bonding potential in proteins. J Mol Biol 1994;238:777-793.

16. Ramakrishnan C, Srinivasan N. Glycyl residues in proteins and peptides: an analysis. Curr Sci 1990;59:851-861.

17. Kabsch W, Sander C. Dictionary of protein secondary structure: pattern recognition of hydrogen-bonded and geometrical features. Biopolymers 1983;22:2577-2637.

18. Lee B, Richards FM. The interpretation of protein structures: estimation of static accessibility. J Mol Biol 1971;55:379-400.

19. Chakravarty S, Varadarajan R. Residue depth: a novel parameter for the analysis of protein structure and stability. Struct Fold Des 1999;7:723-732.

20. Rubin SM, Lee SY, Ruiz EJ, Pines A, Wemmer DE. Detection and characterization of xenon-binding sites in proteins by $129 \mathrm{Xe}$ NMR spectroscopy. J Mol Biol 2002;322:425-440.

21. Alber T, Bell JA, Sun DP, Nicholson H, Wozniak JA, Cook S, Matthews BW. Replacements of Pro86 in phage T4 lysozyme extend an alpha-helix but do not alter protein stability. Science 1988;239:631-635.

22. Yuan HS, Wang SS, Yang WZ, Finkel SE, Johnson RC. The structure of Fis mutant Pro61Ala illustrates that the kink within the long alpha-helix is not due to the presence of the proline residue. J Biol Chem 1994;269:28947-28954.

23. Rudresh, Jain R, Dani V, Mitra A, Srivastava S, Sarma SP, Varadarajan R, Ramakumar S. Structural consequences of replacement of an alpha-helical Pro residue in Escherichia coli thioredoxin. Protein Eng 2002;15:627-633.

24. Chakrabarti P, Chakrabarti S. C-H hydrogen bond O hydrogen bond involving proline residues in alpha-helices. J Mol Biol 1998;284:867-873.

25. Valadone P, Sayle R, Mueller A, Bernstein H. RasTop (version 1.3). http://www.geneinfinity.org/rastop 2000;. 